\title{
Breadth of Tuning and Taste Coding in Mammalian Taste Buds
}

\author{
Seth M. Tomchik, ${ }^{1}$ Stephanie Berg, ${ }^{1}$ Joung Woul Kim, ${ }^{1}$ Nirupa Chaudhari, ${ }^{1,2}$ and Stephen D. Roper ${ }^{1,2}$ \\ ${ }^{1}$ Department of Physiology and Biophysics and ${ }^{2}$ Program in Neurosciences, University of Miami Miller School of Medicine, Miami, Florida 33136
}

\begin{abstract}
A longstanding question in taste research concerns taste coding and, in particular, how broadly are individual taste bud cells tuned to taste qualities (sweet, bitter, umami, salty, and sour). Taste bud cells express G-protein-coupled receptors for sweet, bitter, or umami tastes but not in combination. However, responses to multiple taste qualities have been recorded in individual taste cells. We and others have shown previously there are two classes of taste bud cells directly involved in gustatory signaling: "receptor" (type II) cells that detect and transduce sweet, bitter, and umami compounds, and "presynaptic" (type III) cells. We hypothesize that receptor cells transmit their signals to presynaptic cells. This communication between taste cells could represent a potential convergence of taste information in the taste bud, resulting in taste cells that would respond broadly to multiple taste stimuli. We tested this hypothesis using calcium imaging in a lingual slice preparation. Here, we show that receptor cells are indeed narrowly tuned: $82 \%$ responded to only one taste stimulus. In contrast, presynaptic cells are broadly tuned: $83 \%$ responded to two or more different taste qualities. Receptor cells responded to bitter, sweet, or umami stimuli but rarely to sour or salty stimuli. Presynaptic cells responded to all taste qualities, including sour and salty. These data further elaborate functional differences between receptor cells and presynaptic cells, provide strong evidence for communication within the taste bud, and resolve the paradox of broad taste cell tuning despite mutually exclusive receptor expression.
\end{abstract}

Key words: taste bud; cell type; taste processing; presynaptic cell; receptor cell; coding

\section{Introduction}

A major controversy in taste research has been how the fundamental taste qualities (sweet, bitter, umami, sour, and salty) are encoded in taste buds (e.g., "labeled lines" vs "across-fiber patterns”) (Chandrashekar et al., 2006; Simon et al., 2006). The answer to this controversy in part depends on how broadly "tuned" individual gustatory sensory cells are to different taste stimuli, i.e., whether a single cell responds to one or multiple taste stimuli.

Bitter, sweet, and umami taste qualities are transduced by G-protein-coupled receptors (GPCRs) that signal through a common pathway, including phospholipase C $\beta 2$ (PLC $\beta 2$ ) and the cation channel TRPM5 (transient receptor potential melastatin 5) (Perez et al., 2002; Zhang et al., 2003). Taste GPCRs are expressed in a mutually exclusive manner: cells express receptors for only one taste quality (Nelson et al., 2001). The taste cell (rather than the receptor) determines the perceived quality of the ligand of the receptor (Mueller et al., 2005), and this has been interpreted to mean that taste is encoded by labeled lines (Chandrashekar et al., 2006). However, physiological studies have con-

Received April 24, 2007; revised Aug. 5, 2007; accepted Aug. 8, 2007.

This work was supported by National Institutes of Health (NIH)-National Institute on Deafness and Other Communication Disorders Grants R01DC000374, R01DC007630 (S.D.R.), and R01DC006308 (N.C.) and NIH-National Institute of Neurological Disorders and Stroke Training Grant T32NS007044 (S.M.T.). We thank Drs. Y. Maruyama and A. Caicedo for technical assistance. Transgenic GAD-GFP mice were a kind gift from Dr. Z. Josh Huang (Brandeis University, Waltham, MA).

Correspondence should be addressed to Dr. Stephen D. Roper, Department of Physiology and Biophysics (4065 Rosenstiel Medical Science Building), University of Miami Miller School of Medicine, 1600 N.W. 10th Avenue, Miami, FL33136. E-mail: sroper@med.miami.edu.

DOI:10.1523/JNEUROSCI.1863-07.2007

Copyright $\odot 2007$ Society for Neuroscience ～0270-6474/07/2710840-09\$15.00/0 sistently demonstrated that single taste cells and afferent fibers frequently respond to two or more taste qualities, challenging the notion of labeled lines. Microelectrode recordings, patch-clamp recordings, and calcium imaging all demonstrated that a significant proportion of taste cells respond to multiple taste stimuli (Sato and Beidler, 1997; Gilbertson et al., 2001; Caicedo et al., 2002; Yoshida et al., 2006). A resolution proposed recently (Roper, 2006; Huang et al., 2007) is that cell-to-cell communication and signal integration within the taste bud distributes the signals and results in individual taste cells responding more broadly than their receptor complement would suggest.

It is now widely recognized that there are two types of taste cells in the taste bud: "receptor" (type II) cells and "presynaptic" (type III) cells (Yee et al., 2001; Clapp et al., 2006; DeFazio et al., 2006). Receptor cells express taste GPCRs and transduce sweet, bitter, or umami taste stimuli, but they do not form conventional synapses. Conversely, presynaptic taste cells do not have GPCRs for tastants but instead express synapse-related proteins such as synaptosome-associated protein of $25 \mathrm{kDa}$ (SNAP-25) and contain synaptic specializations (Yang et al., 2000). Receptor cells secrete ATP, which stimulates presynaptic cells (Roper, 2006; Huang et al., 2007) and sensory afferent fibers (Finger et al., 2005). Isolated receptor cells respond to bitter or sweet tastants but presynaptic cells do not (DeFazio et al., 2006). In summary, the two different types of chemosensory taste cells have distinctly different properties and presumably different functions.

In situ, receptor cells presumably respond directly to bitter, sweet, and umami tastants and may in turn stimulate presynaptic cells, providing an indirect route for presynaptic cells to respond to those taste stimuli. Such intragemmal communication, if it 
occurs, would resolve the aforementioned paradox of narrowly tuned molecular receptor expression versus multiple sensitivity. Receptor cells might be narrowly tuned, whereas presynaptic cells receiving converging input from receptor cells would be more broadly responsive to taste stimulation. We directly tested this hypothesis using calcium imaging in a semi-intact lingual slice preparation.

\section{Materials and Methods}

Tissue preparation and functional imaging. All experimental procedures were approved by the University of Miami Animal Care and Use Committee. Animals were killed by exposure to $\mathrm{CO}_{2}$ followed by cervical dislocation. Three lines of mice were used in these experiments: C57BL/6 mice (wild-type), transgenic mice expressing enhanced green fluorescent protein (GFP) under control of the PLC $\beta 2$ promoter (PLC $\beta 2-G F P$ ) (Kim et al., 2006), and transgenic mice expressing GFP under the GAD67 promoter (GAD-GFP) (Chattopadhyaya et al., 2004).

We prepared lingual slices containing the vallate papilla from adult mice ( $\geq 8$ weeks old) and loaded taste cells with a calcium indicator dye (Caicedo et al., 2000, 2002). Briefly, a fluorescent $\mathrm{Ca}^{2+}$ indicator dye was injected iontophoretically through a $40-\mu \mathrm{m}$-tip glass micropipette into the crypt surrounding the vallate papilla. We loaded taste cells with Calcium Green-1 dextran (CaGD) ( $1 \mathrm{~mm}$ in $\mathrm{H}_{2} \mathrm{O}$, molecular weight of $10,000 \mathrm{kDa}$; Invitrogen, Carlsbad, CA) (wild-type mice) or Calcium Orange (1 mM in $\mathrm{H}_{2} \mathrm{O}, 1087 \mathrm{kDa}$; Invitrogen) (transgenic mice expressing GFP). Dye-loaded tissue was cut into $100 \mu \mathrm{m}$ sections with a vibratome (VT1000S; Leica, Nussloch, Germany). Slices containing taste buds were mounted on a glass coverslip coated with Cell-Tak (BD Biosciences, San Jose, CA), placed in a recording chamber, and superfused with Tyrode's solution $\left(30^{\circ} \mathrm{C}\right)$ at a rate of $1 \mathrm{ml} / \mathrm{min}$. Single glass micropipettes ("puffer" pipettes, $2 \mu \mathrm{m}$ tip diameter) were used to deliver taste stimuli directly to the taste pores of taste buds in the lingual slice. Stimuli were ejected for $2 \mathrm{~s}$ with air pressure (1-5 psi) (Picospritzer; Medical Systems, Greenvale, NY).

Lingual slices containing dye-loaded taste cells were viewed with a scanning laser confocal microscope using argon and krypton lasers (Fluoview; Olympus Optical, Thornwood, NY). Images were captured at $1.1 \mathrm{~s}$ intervals for tastant-evoked responses or $5 \mathrm{~s}$ intervals for bathapplied $\mathrm{KCl}$ responses. We have presented fluorometric signals as relative fluorescence change: $\Delta F / F=\left(F-F_{0}\right) / F_{0}$. Correction for photobleaching was applied when necessary (Caicedo et al., 2000). Taste cells were stimulated repeatedly with a selection of prototypic taste stimuli (see next section). We could record responses for up to $8 \mathrm{~h}$. At the end of any given recording session, the taste stimulus that evoked the largest response was reapplied to test that the preparation was still viable. Only data from cells that were still responsive at the end of the experiment were analyzed in this report.

Reagents and solutions. Unless otherwise indicated, chemicals were purchased from Sigma (St. Louis, MO). Slices were bathed in normal Tyrode's solution with the following composition (in $\mathrm{mM}$ ): $130 \mathrm{NaCl}, 5 \mathrm{KCl}, 8 \mathrm{CaCl}_{2}$, $1 \mathrm{MgCl}_{2}, 10 \mathrm{HEPES}, 10$ glucose, 10 sodium pyruvate, and $5 \mathrm{NaHCO}_{3}$, adjusted to $\mathrm{pH} 7.4$ (318-323 mOsm). Calcium was elevated ( $8 \mathrm{~mm}$ ) to enhance responses and improve the signal-to-noise ratio. High- $\mathrm{K}^{+}$Tyrode's solution contained the same constituents with the exception that $50 \mathrm{~mm} \mathrm{KCl}$ was equimolar substituted for $\mathrm{NaCl}$ (final concentrations of $50 \mathrm{~mm} \mathrm{KCl}$ and 85 $\mathrm{mm} \mathrm{NaCl}$ ). Seven taste stimuli were used in these experiments: SC45647 (2-[[[[4-(aminomethyl)phenyl] amino]-[[(1R)-1-phenylethyl] amino]methyl] amino] ethane-1,1-diol) (gift from Dr. G. Dubois, Coca Cola, Atlanta, GA), sucrose, cycloheximide, denatonium, monopotassium L-glutamate (MPG), $\mathrm{NaCl}$, and citric acid. Inosine $5^{\prime}$-monophosphate (IMP) at $1 \mathrm{~mm}$ was added to all MPG solutions to enhance umami taste responses.

To locate taste-responsive cells, we used a search stimulus containing a mixture of $30 \mu \mathrm{M}$ cycloheximide, $200 \mu \mathrm{M}$ denatonium, $100 \mu \mathrm{M}$ SC45647, $200 \mathrm{~mm}$ MPG, and $1 \mathrm{~mm}$ IMP. Once a responsive cell or group of cells was found, all cells in the area were tested for sensitivity to individual taste stimuli, presented in random order. Individual tastants were $30 \mu \mathrm{M} \mathrm{cy-}$ cloheximide, $200 \mu \mathrm{M}$ denatonium, $500 \mathrm{~mm}$ sucrose, $100 \mu \mathrm{M}$ SC45647, $200 \mathrm{~mm}$ MPG plus $1 \mathrm{~mm} \mathrm{IMP,} 500 \mathrm{~mm} \mathrm{NaCl}$, and $100 \mathrm{~mm}$ citric acid.
These concentrations were selected based on previous studies showing that they produce robust and reliable calcium responses (Caicedo et al., 2002; Richter et al., 2003; Maruyama et al., 2006). All tastant solutions (except citric acid) contained $2 \mu \mathrm{M}$ fluorescein to monitor stimulus application, duration, and concentration. Citric acid solutions contained $500 \mu \mathrm{M}$ Lucifer yellow. All tastants were dissolved in normal Tyrode's solution.

Immunostaining. Circumvallate papillae from GAD-GFP mice were fixed, cryosectioned at $20 \mu \mathrm{m}$, and immunostained following the procedures of DeFazio et al. (2006) except with the addition of a 1\% Triton $\mathrm{X}-100$ permeabilization step after fixation. The following antibodies were used: anti-PLC $\beta 2$ (1:1000; sc-206; Santa Cruz Biotechnology, Santa Cruz, CA), anti-SNAP-25 (1:1000-1:4000; AB1762; Chemicon, Temecula, CA), and anti-serotonin (5-HT) (1:500; MAB352; Chemicon). For anti-serotonin immunostaining, mice were preinjected with $200 \mu \mathrm{l}$ of Tyrode's solution containing $2 \mathrm{mg}$ of the serotonin precursor 5-hydroxytryptophan $1 \mathrm{~h}$ before the animals were killed. We used Alexa Fluor 594 goat IgG secondary antibodies (1:500; A21096 or A21207; Invitrogen). Negative controls were processed in parallel in every experiment with primary antibodies omitted. No nonspecific fluorescence was detected (see Fig. $3 H$ ). Images were obtained with an Olympus Optical laser-scanning confocal microscope using Fluoview software.

To immunostain lingual slices after calcium imaging (Maruyama et al., 2006), we used anti-PLC $\beta 2$ (1:1000) or anti-SNAP-25 (1:500) rabbit primary antibodies (as above) for $3 \mathrm{~d}$ at room temperature, followed by washing, and then Alexa Fluor 660 goat anti-rabbit IgG (1:500; A-21073; Invitrogen) for 1-3 d, again at room temperature. In several experiments, GFP fluorescence was enhanced by immunostaining with chicken antiGFP (1:500; GFP-1020; Aves Labs, Tigard, OR), followed by Alexa Fluor 488 goat anti-chicken IgG (1:500; A-11039; Invitrogen). Confocal images were matched and overlaid with those obtained during the functional $\mathrm{Ca}^{2+}$ imaging.

Data analysis. Baseline fluorescence was measured in the $5 \mathrm{~s}$ interval before each stimulus application. We only included data from preparations in which the baseline fluorescence fluctuated less than $\sim 2 \% \Delta F / F$. Calcium transients in taste cells were scored blindly with a customwritten Excel Visual Basic macro that displayed each trace on the screen and allowed the reviewer to visually identify it as either a response or a failure. To determine whether each trace represented a true response, we used the criteria of Caicedo et al. (2000) as follows. We scored positive responses if (1) the peak $\Delta F / F$ was at least two times the baseline fluctuation for that cell, (2) responses could be elicited at least twice in the same cell by the same stimulus and appeared approximately of the same amplitude, and (3) responses were not observed in all dye-loaded cells in the field of view (i.e., signals were not caused by a stimulus artifact). The peak $\Delta F / F$ was quantified for each response, and a metric of breadth of tuning, entropy $(H)$, was calculated for each cell (Smith and Travers, 1979). Responses to the five taste qualities were compared between groups of cells using Fisher's exact test. Data analyses were performed in Microsoft (Seattle, WA) Excel and R (http://www.R-project.org).

\section{Results}

\section{Narrowly tuned and broadly responsive cells in taste buds}

The major goal of these experiments was to test the hypothesis that presynaptic (type III) cells in taste buds receive converging input from receptor (type II) cells and consequently are more broadly tuned to taste quality than are receptor cells. To test this hypothesis, we used functional imaging with CaGD in lingual slice preparations of mouse vallate papillae. We tested taste cell responses to seven different taste stimuli applied focally to the taste pore and to depolarization with bath-applied $\mathrm{KCl}(50 \mathrm{~mm})$. [Only presynaptic cells have voltage-sensitive calcium channels and show calcium influx when depolarized (Clapp et al., 2006; DeFazio et al., 2006)]. The seven taste stimuli that we used included at least one tastant from each of the five basic qualities: (1) bitter: cycloheximide or denatonium; (2) sweet: sucrose or SC45647; (3) umami: MPG plus IMP; (4) salty: $\mathrm{NaCl}$; (5) sour: 
citric acid. Responses to these taste stimuli and to $\mathrm{KCl}$ depolarization are illustrated in Figure 1.

Of 44 tastant-responsive cells recorded in this initial analysis, $61 \%(n=27)$ responded to a single quality (Fig. 1A), whereas the remaining cells responded to two or more qualities (Fig. $1 B$ ). These numbers agree well with previous findings on the multiple taste sensitivity of mouse vallate taste cells [55\% (Caicedo et al., 2002)] and mouse fungiform taste cells [67\% (Yoshida et al., 2006)].

To determine the cell type for each recorded cell, we examined their responses to depolarization with $\mathrm{KCl}$. Presynaptic (type III) cells can be distinguished by their pronounced depolarization-dependent calcium influx (Fig. 1B) (DeFazio et al., 2006; Huang et al., 2007). Taste-responsive cells that did not exhibit depolarization-evoked calcium responses were provisionally classified as receptor (type II) cells. In the set of 44 tastantresponsive cells, we identified 14 presynaptic cells and 30 receptor cells. Cells classified as presynaptic were broadly responsive to taste stimuli; $86 \%$ (12 of 14) responded to two or more taste qualities. In contrast, cells provisionally identified as receptor cells were more narrowly responsive. Eighty-three percent ( 25 of 30 ) responded to only one taste quality. The breadth of tuning differed significantly between putative receptor cells and presynaptic cells (Fisher's exact test, $p<0.001$ ).

To identify cell types more confidently, we immunostained lingual slices for cell-specific markers after calcium imaging. Specifically, we immunostained for PLC $\beta 2$ (receptor cells) or SNAP-25 (presynaptic cells) (Fig. 2). We were able to relocate 18 of the cells that had been provisionally identified as receptor cells in physiological recordings. Of these, 11 were immunoreactive for PLC $\beta 2$, confirming that indeed they were receptor cells (Fig. $2 A-C$ ) (supplemental Table 3, available at www.jneurosci.org as supplemental material). Each of the PLC $\beta 2$-positive cells was narrowly responsive to taste stimuli and responded to only one stimulus (Fig. $2 D$, cell 2). The remaining seven provisional receptor cells failed to immunostain sufficiently strongly with antiPLC $\beta 2$ to identify them with confidence. Presynaptic (i.e., $\mathrm{KCl}$ responsive) cells in the same slices (Fig. $2 A-D$, cell 1) were broadly tuned and lacked PLC $\beta 2$ immunostaining.

Post hoc immunostaining for SNAP-25 to identify presynaptic cells was more problematic in thick sections attributable to the uneven cellular distribution of reaction product and generally weak immunofluorescence. Consequently, we were able to confidently correlate SNAP-25 immunoreactivity with $\mathrm{KCl}$ responses in only one cell (Fig. $2 E-G$ ). This cell had responded broadly to the panel of tastants and exhibited a robust depolarization-evoked calcium response (Fig. $2 H$ ). None of the $\mathrm{KCl}$-unresponsive cells from these slices was immunopositive for SNAP-25.

In brief, for this initial series, we were able to distinguish receptor cells and presynaptic cells using $\mathrm{KCl}$ sensitivity, validate a subset of these cells by post hoc immunostaining, and show that they differed in the breadth of their response to a panel of taste stimuli.

\section{Using transgenic mice to identify receptor cells and presynaptic cells}

Next, we designed additional methods to distinguish receptor (type II) cells and presynaptic (type III) cells using independent methods from those described above. To identify receptor cells, we used transgenic mice expressing GFP on the PLC $\beta 2$ promoter (Kim et al., 2006). Taste receptor cells in these mice are readily visible in living slice preparations viewed with fluorescence microscopy.

To identify presynaptic (type III) cells, we used GAD-GFP mice (Chattopadhyaya et al., 2004). [We previously showed that presynaptic cells express glutamic acid decarboxylase (DeFazio et al., 2006)]. To verify that GFP fluorescence was reliably and selectively expressed in presynaptic taste cells in these mice, we immunostained $20 \mu \mathrm{m}$ cryostat sections of vallate papillae from GAD-GFP mice for SNAP-25 (Fig. 3A-D,L). Of 647 counted cells, 72\% $(n=468)$ expressed both GFP and SNAP-25, $1 \%(n=$ 8) expressed GFP alone without SNAP-25, and 26\% $(n=171)$ expressed SNAP-25 but not GFP. In brief, 98\% (468 of 476) of GFP-positive cells expressed SNAP-25 and therefore GFP could be used as a reliable marker of presynaptic cells in the lingual slice (Table 1).

Parenthetically, presynaptic taste cells are serotonergic and release 5-HT during stimulation (Yee et al., 2001; Huang et al., 2005, 2007; DeFazio et al., 2006). Thus, we also immunostained taste buds from GAD-GFP mice for the presence of 5-HT (Fig. $3 E-G, L)$. Of 432 counted cells, $77 \%(n=334)$ expressed both GFP and 5-HT, fewer than 1\% expressed GFP but not 5-HT $(n=$ 2 ), and $22 \%(n=96)$ expressed only 5 -HT. Thus, $>99 \%$ (334 of 336) of GFP-positive cells in GAD-GFP mice are serotonergic, demonstrating that GAD and serotonin are present together in presynaptic taste cells (Table 2).

Last, because these findings show GFP is reliably expressed in presynaptic cells, one would not expect to find GFP in cells expressing PLC $\beta 2$ (i.e., in receptor cells). We tested this by immu- 

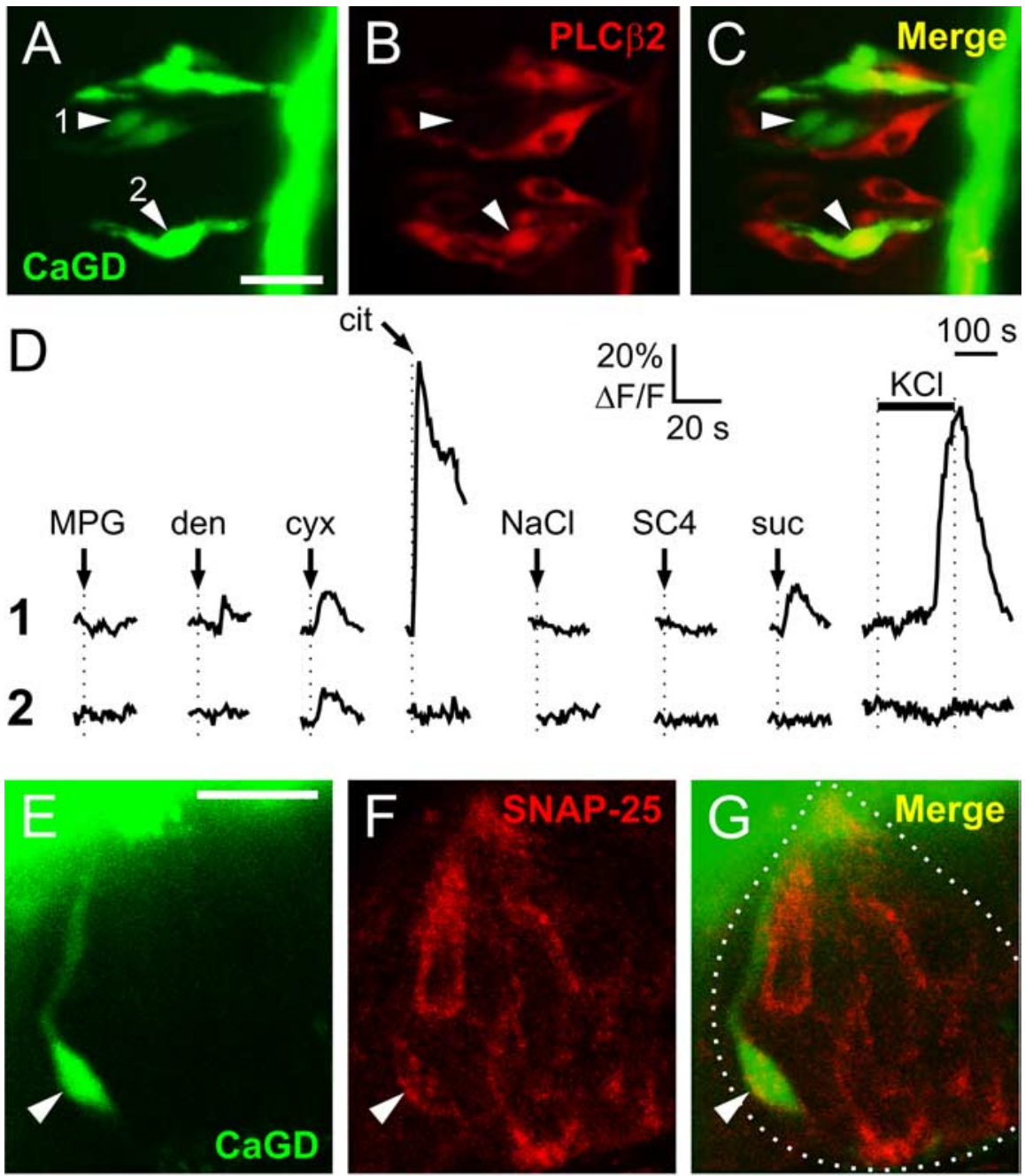

$\mathrm{H}$

cit
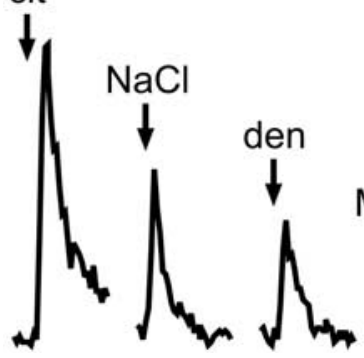

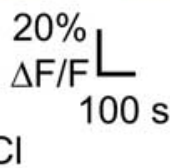

$100 \mathrm{~s}$

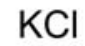

Figure 2. Post hoc immunostaining of lingual slices to identify receptor (type II) and presynaptic (type III) cells. Receptor cells are identified by the presence of PLC $\beta 2$ immunostaining and presynaptic cells by SNAP-25 immunopositivity. $\boldsymbol{A}-\boldsymbol{C}$, Confocal micrographs showing two adjacent taste buds in a lingual slice. $\boldsymbol{A}$, Many cells are loaded with CaGD (green). $\boldsymbol{B}$, After recording taste-evoked responses, the tissue was immunostained for PLC $\beta 2$ (red) to identify receptor cells. Arrowheads point to two tastant-responsive taste cells $(1,2)$. Cell 1 was PLC $\beta 2$ negative, whereas cell 2 (and several other cells in each taste bud) were immunoreactive for PLC $\beta 2$. C, Merged view. Scale bar, $20 \mu \mathrm{m}$. D, Calcium imaging traces of the taste cells highlighted in $\boldsymbol{A}-\boldsymbol{C}$. Cell 1 responded when depolarized with $50 \mathrm{~mm} \mathrm{KCl}$, demonstrating that it was a presynaptic cell. Cell 1 also responded broadly to taste stimuli, responding to denatonium (den), cycloheximide (cyx), citric acid (cit), and sucrose (suc). In contrast, cell 2 did not respond to $\mathrm{KCl}$ depolarization. Furthermore, cell 2 responded only to cycloheximide. The $20 \mathrm{~s}$ calibration bar applies to all taste responses; the 100 s calibration bar applies only to the $\mathrm{KCl}$-evoked responses. $\boldsymbol{E}-\mathbf{G}$, Confocal micrographs showing a taste bud in a lingual slice immunostained for SNAP-25 (red) to identify presynaptic cells. The taste bud is outlined with a dotted white line in G. Scale bar, $20 \mu \mathrm{m}$. $\boldsymbol{H}$, Calcium imaging of the taste cell identified (arrowhead) in $\boldsymbol{E}-\boldsymbol{G}$. This cell responded to $\mathrm{KCl}$ depolarization and to several taste stimuli, including citric acid, $\mathrm{NaCl}$, denatonium, cycloheximide, and SC45647. The 20 s calibration bar applies to all tastant responses, and the $100 \mathrm{~s}$ calibration bar applies only to the $\mathrm{KCl}$-evoked response. MPG, $200 \mathrm{~mm}$ monopotassium L-glutamate plus 1 mм IMP; den, 200 mm denatonium; cyx, $30 \mu$ m cycloheximide; cit, 100 mm citric acid; $\mathrm{NaCl}, 500$ mм NaCl; SC4, $100 \mu$ m SC45647; suc, 500 mм sucrose; $\mathrm{KCl}, 50 \mathrm{~mm} \mathrm{KCl}$.

nostaining taste buds from GAD-GFP mice for PLC $\beta 2$ (Fig. 3I-L). Of 246 cells counted, no GAD-GFP-positive cells expressed PLC $\beta 2,43 \%(n=106)$ expressed only PLC $\beta 2$, whereas 57\% $(n=140)$ expressed only GFP (Table 3 ).

Collectively, the above data indicate that GFP is faithfully expressed in presynaptic (type III) cells and not in receptor (type II) cells in GAD-GFP mice.

Using transgenic mice to test breadth of tuning in receptor and presynaptic cells Taste cells in the above two strains of transgenic mice were loaded with Calcium Orange to measure taste-evoked $\mathrm{Ca}^{2+}$ responses. We identified and recorded responses from 26 receptor cells in PLC $\beta 2-$ GFP mice (Fig. $4 A-D$ ) and 44 presynaptic cells in GAD-GFP mice. In PLC $\beta 2-G F P$ mice, cells that did not express GFP but exhibited depolarization-evoked calcium responses were classified as presynaptic cells, as before. In GAD-GFP mice, we also used post hoc immunohistochemistry for PLC $\beta 2$ to identify additional receptor cells.

Taste cells in the two transgenic mouse strains responded similarly to their counterparts in wild-type mice. There was no difference in the breadth of tuning or pattern of responses to the taste stimuli between receptor cells identified in wild-type versus PLC $\beta 2-$ GFP mice (Fisher's exact tests, $p>0.05$ ). Presynaptic cells identified in wild-type mice also responded similarly to presynaptic cells in GAD-GFP mice (Fisher's exact tests, $p>0.05$ ). Hence, we pooled the data for additional analysis. The complete response profiles of all 114 cells (56 receptor cells, 58 presynaptic cells) are shown in supplemental Tables 1 and 2 (available at www.jneurosci.org as supplemental material).

The combined dataset confirmed that receptor cells are considerably more narrowly responsive to taste stimuli than presynaptic cells. Eighty-two percent (46 of $56)$ of receptor cells responded to only one taste quality (see Figs. 5A,6) (supplemental Table 1, available at www.jneurosci.org as supplemental material). On average, a receptor cell responded to $1.21 \pm 0.07$ qualities (mean \pm SEM). No receptor cell responded to more than three different taste qualities. In contrast, the majority of presynaptic cells (83\%; 48 of 58 ) responded to two or more taste qualities (mean of $2.86 \pm 0.38$ ). Indeed, we observed some presynaptic cells responding to all five (Fig. 5A) (supplemental Table 2, available at www.jneurosci.org as supplemental material). Presynaptic cells were 

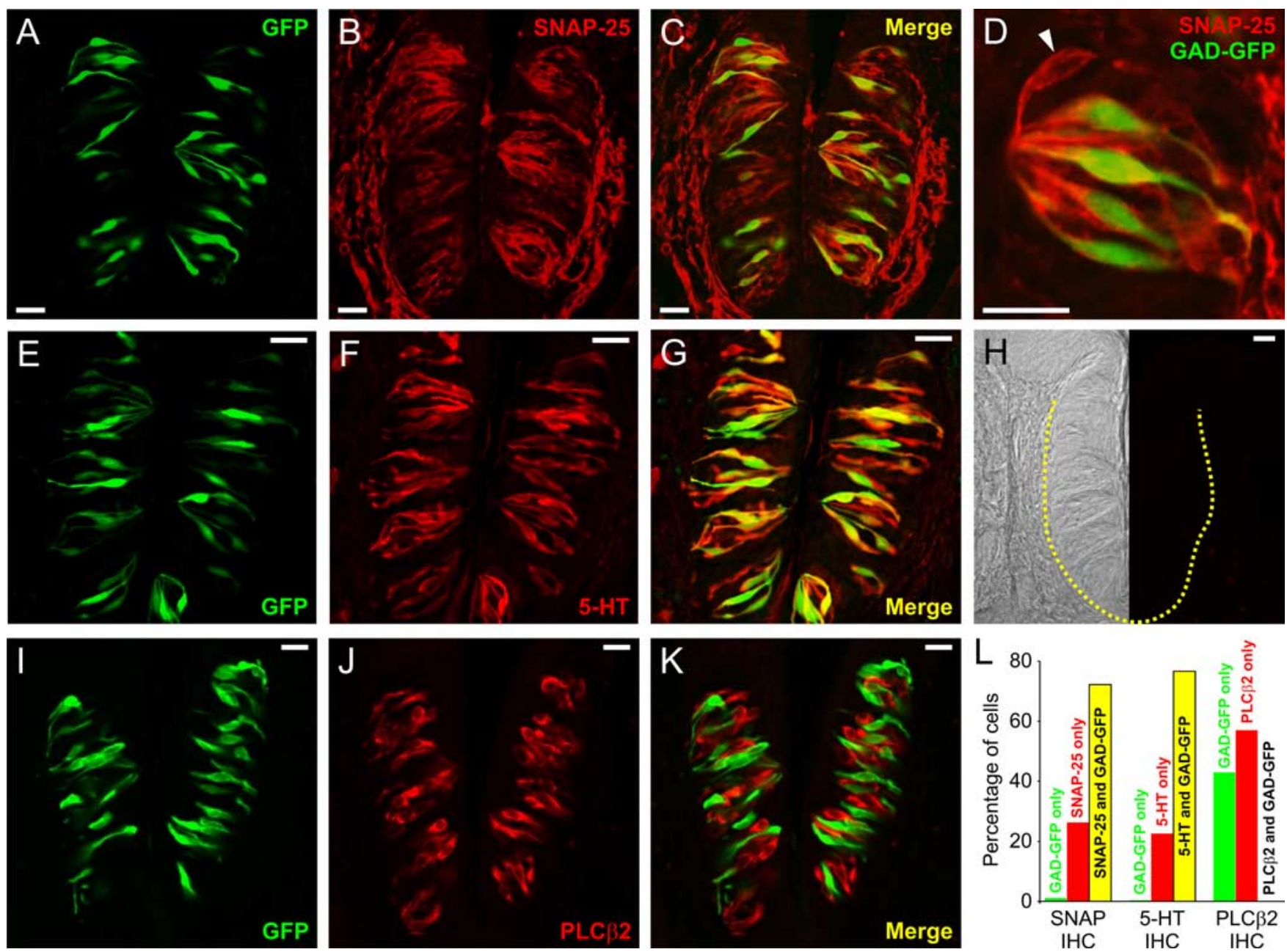

Figure 3. GFP identifies presynaptic (type III) cells in GAD-GFP mice. Confocal micrographs of $20 \mu \mathrm{m}$ cryosections of vallate taste buds from GAD-GFP mice. A-C SNAP-25 immunostaining, showing immunolabel (red) in taste cells expressing GFP (green) and in nerve fibers. The merged image is shown in C. D, Higher magnification of one taste bud with merged SNAP-25 immunostaining and GFP fluorescence. Four cells in this taste bud expressed both GFP and SNAP-25; one cell expressed only SNAP-25 (arrowhead). E-G, 5-HT immunostaining (red). GFP (green) is expressed in many but not all serotonergic taste cells. $\boldsymbol{H}$, Negative control, processed in parallel without primary antibodies, showing no detectable immunofluorescence (GFP fluorescence is not shown). The boundary of the epithelium containing taste cells is outlined; a micrograph using Nomarski differential contrast optics is overlaid on the left side only. I- $\boldsymbol{K}$, PLC $\beta 2$ immunostaining (red). $\boldsymbol{K}$, Merged image. Note that GFP (green) is not present in PLC $\beta 2$-immunoreactive taste cells. L, Quantification of results. Each set of three columns shows the percentage of taste cells expressing GAD-GFP only (green), immunostaining only (red) (SNAP-25, 5-HT, or PLC $\beta 2$ ), or the presence of both GAD-GFP fluorescence and immunostaining (yellow). IHC, Immunohistochemistry. Scale bars, $20 \mu \mathrm{m}$.

significantly more broadly responsive than receptor cells (Fisher's exact test, $p<0.001$ ).

In addition to examining the number of stimuli that elicited responses, we quantified the magnitude (average peak $\Delta F / F$ across stimulus repetitions) of each response for every cell (supplemental Tables 1, 2, available at www.jneurosci.org as supplemental material). These values were used to calculate a measure of breadth of tuning, called entropy $(H)$ (Smith and Travers, 1979). $H$ varies between 0.0 (very narrow breadth of tuning) to 1.0 (broadly tuned, i.e., equally responsive to all stimuli). Receptor cells had a mean $H$ of $0.07 \pm 0.02$, whereas presynaptic cells

Table 1. GAD-GFP taste cells express SNAP-25, a marker for presynaptic (type III) taste cells

\begin{tabular}{cccc}
\hline No. of cells (647 total) & Proportion of cells & GFP & SNAP-25 \\
\hline 8 & $1 \%$ & + & - \\
171 & 26 & - & + \\
468 & 72 & + & +
\end{tabular}

Vallate taste buds from GAD-GFP mice were immunostained for SNAP-25 (a marker for presynaptic cells) and examined for coexpression of GFP and SNAP-25. Of 476 cells with GFP, 468 (98\%) also expressed SNAP-25. №. Number. had a significantly higher $H$ of $0.47 \pm 0.04$ (Student's $t$ test, $p<$ 0.001 ) and thus were more broadly tuned. Supplemental Table 4 (available at www.jneurosci.org as supplemental material) presents the response profiles of presynaptic cells, classified by their optimal qualities.

Sweet, bitter, and umami stimuli are transduced by receptor cells, whereas presynaptic cells do not express the corresponding taste GPCRs (DeFazio et al., 2006). Nonetheless, we found that presynaptic cells responded to bitter sweet and umami compounds. Indeed, there was no significant difference between the distributions of receptor and presynaptic cells responding to sweet, bitter, and umami stimuli (Fig. 5B). Presynaptic cell re-

Table 2. GAD-GFP taste cells are serotonergic

\begin{tabular}{cccc}
\hline No. of cells (432 total) & Proportion of cells & GFP & 5 -HT \\
\hline 2 & $1 \%$ & + & - \\
96 & 22 & - & + \\
334 & 77 & + & + \\
\hline
\end{tabular}

Vallate taste buds from GAD-GFP mice were immunostained for 5 -HT and examined for coexpression of GFP and 5-HT. of 334 cells that expressed GFP, 332 (99\%) were immunopositive for 5-HT. No., Number. 
Table 3. GAD-GFP taste cells are distinct from cells expressing PLC $\beta 2$ (i.e., receptor cells)

\begin{tabular}{llcc}
\hline No. of cells (246 total) & Proportion of cells & GFP & PLC $\beta 2$ \\
\hline 140 & $57 \%$ & + & - \\
106 & 43 & - & + \\
0 & 0 & + & + \\
\hline
\end{tabular}

Taste buds from GAD-GFP mice were immunostained for PLC $\beta 2$ (a marker for receptor cells) and examined for coexpression of GFP and PLC $\beta 2$. Of 246 cells that expressed GFP, none were immunopositive for PLC $\beta 2$.

sponses might be explained by cell-to-cell communication within the taste bud and convergence of inputs from receptor onto presynaptic cells (Huang et al., 2007). Some taste cells responded to stimuli of opposing appetitive quality (e.g., sweet and bitter; umami and bitter). Intriguingly, the majority of these (11 of 13) were presynaptic cells.
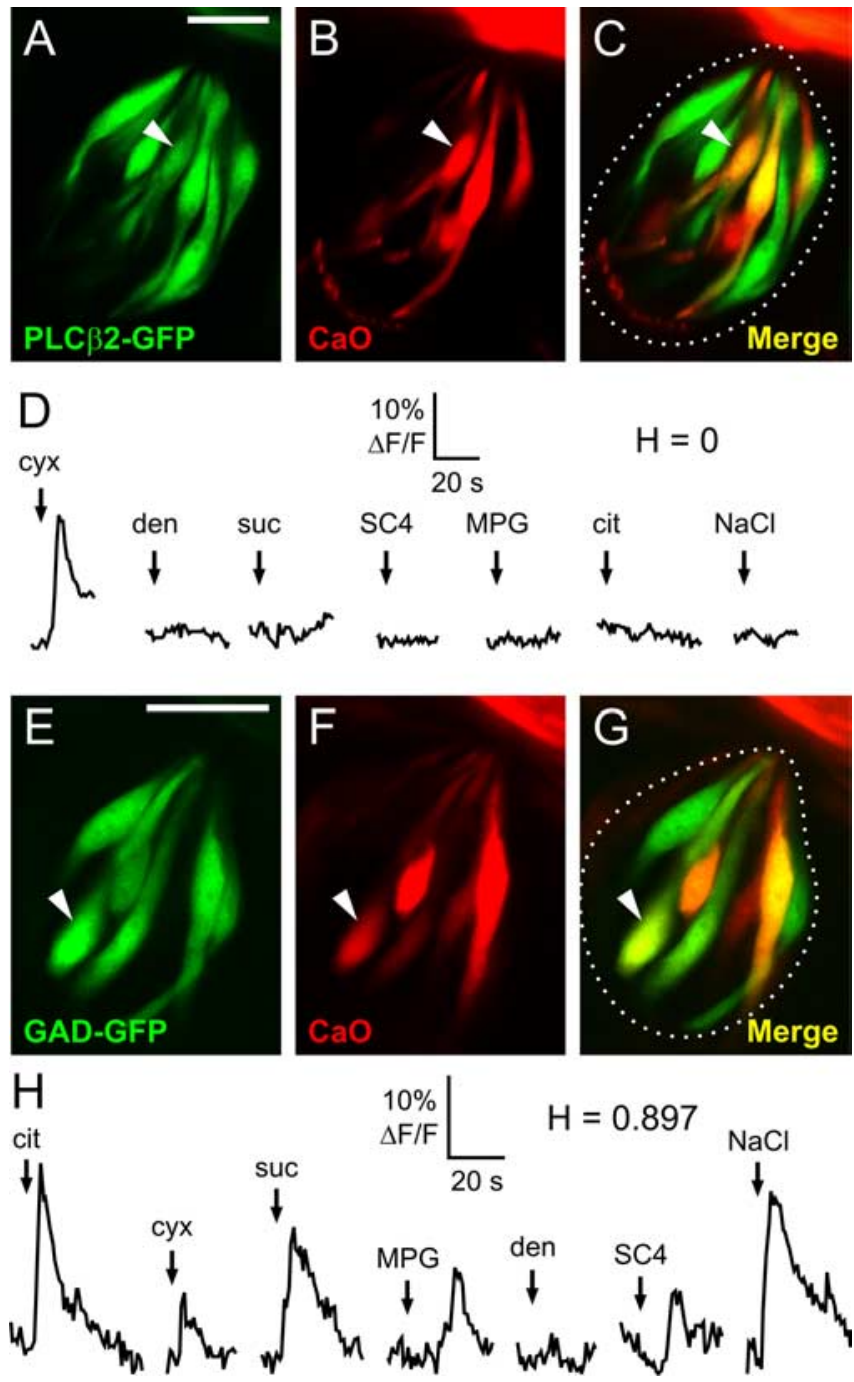

Figure 4. Calcium imaging in lingual slices from transgenic mice shows that receptor (type II) cells are narrowly responsive and presynaptic (type III) cells are broadly responsive to taste stimulation. $\boldsymbol{A}-\boldsymbol{C}$, Confocal micrographs of a vallate taste bud from a PLC $\beta 2-$ GFP mouse. $\boldsymbol{A}$, Many cells express PLC $\beta 2-G F P$ (green). $\boldsymbol{B}$, Some taste cells are loaded with the functional imaging dye, Calcium Orange ( $\mathrm{Ca} 0$ ) (red). The taste bud is outlined with a dotted line. $C$, Merged view. D, Responses of PLC $\beta 2-G F P$ cell (i.e., receptor cell) shown in $\boldsymbol{A}-\boldsymbol{C}$ (arrowhead). This cell responded only to cycloheximide. $\boldsymbol{E}-\boldsymbol{G}$, Confocal micrographs of a vallate taste bud from a GAD-GFP mouse. The taste bud is outlined in $\boldsymbol{G}$. $\boldsymbol{H}$, Responses of the GAD-GFP-positive (presynaptic) cell shown in $\boldsymbol{E}-\boldsymbol{G}$ (arrowhead). This cell responded to citric acid, cycloheximide, sucrose, MPG, SC45647, and NaCl. Abbreviations as in Figure 2. Scale bars, $20 \mu \mathrm{m}$.
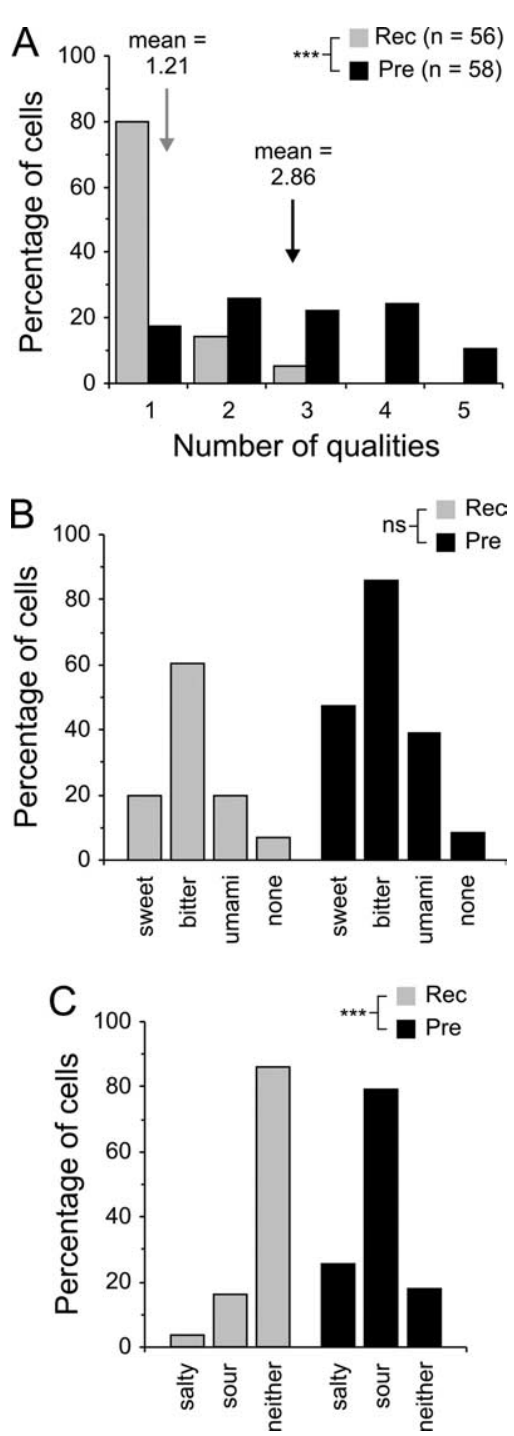

Figure 5. Summary of data from wild-type and transgenic mice showing range of taste responses in receptor and presynaptic taste cells. $\boldsymbol{A}$, Percentage of receptor (Rec) and presynaptic (Pre) cells responding to one or more taste qualities (i.e., sweet, bitter, umami, sour, and salty). There was a significant difference in the incidences of responses to these taste qualities between receptor cells and presynaptic cells (Fisher's exact test, ${ }^{* * *} p<0.001$ ). B, Percentage of receptor and presynaptic cells responding to bitter, sweet, and umami taste qualities. There was no significant difference between receptor and presynaptic cells in the incidence of responses to these taste qualities (Fisher's exact test, $p=0.47$; NS). C, Percentage of receptor and presynaptic cells that responded to sour and salty taste qualities. There was a significant difference between the proportion of receptor and presynaptic cells responding to these taste qualities (Fisher's exact test, ${ }^{* * *} p<0.001$ ).

Presynaptic cells respond to sour and salty stimuli

Salty and sour stimuli are likely transduced by different cells than are bitter, sweet, and umami tastants because they are not believed to be transduced through cells expressing G-proteincoupled taste receptors and PLC $\beta 2$ (Huang et al., 2006; Ishimaru et al., 2006; Lopez-Jimenez et al., 2006). It is not confidently known which class of taste cells (i.e., receptor, presynaptic) is involved in salty and sour taste transduction. Therefore, we analyzed which taste cells responded to sour and salty tastants. There was a significant difference between receptor (type II) cells and presynaptic (type III) cells in their responses to sour (citric acid) and to salty $(\mathrm{NaCl})$ stimuli (Fisher's exact test, $p<0.001$ ) (Fig. $5 C)$. The majority of receptor cells ( $82 \%$; 46 of 56 cells) did not respond to either sour or salty stimuli. In contrast, the majority of 
presynaptic cells (81\%; 47 of 58 cells) responded to citric acid. Presynaptic cells were also more frequently sensitive to $\mathrm{NaCl}(26 \% ; 15$ of 58 cells) than were receptor cells $(2 \% ; 1$ of 56 cells). Among presynaptic cells, the salt-sensitive cells were a subset of acid-sensitive cells. Of the few receptor cells that responded to acid or salty tastes, none responded to both. In summary, presynaptic cells overwhelmingly responded to citric acid and also responded more frequently to $\mathrm{NaCl}$ than receptor cells.

Presynaptic cells frequently exhibited large responses to citric acid (Fig. $4 \mathrm{H}$ and supplemental Table 2, available at www. jneurosci.org as supplemental material). The average $\Delta F / F$ of citric acid responses in presynaptic cells was $24.7 \%$ compared with $16.3 \%$ (cycloheximide), $14.7 \%$ (denatonium), $13.7 \%(\mathrm{NaCl}), 12.5 \%$ (sucrose), $11.5 \%$ (MPG), and $11.3 \%$ (SC45647). Large citric acid responses were not observed in receptor cells. Among the few receptor cells that responded to citric acid, the average response was $11.3 \% \Delta F / F$. Relative magnitudes of the responses to the seven stimuli are plotted for each cell in Figure 6. Many of the presynaptic cells responded maximally to citric acid and also had large responses to the bitter stimulus cycloheximide. Calcium responses evoked by sweet stimuli were typically smaller.

\section{Discussion}

The present data begin to illustrate how gustatory signals are encoded within taste buds and support the concept of cell-cell communication within taste buds (Roper, 1992, 2006; Herness et al., 2005) (Fig. 7). The data also resolve the apparent discrepancy between the nonoverlapping molecular expression patterns of taste receptors (Chandrashekar et al., 2006; Simon et al., 2006) and the broad functional tuning of taste bud cells (Sato and Beidler, 1997; Gilbertson et al., 2001; Caicedo et al., 2002; Yoshida et al., 2006). Receptor (type II) cells are narrowly tuned to qualities transduced by the G-protein-coupled taste receptors $T_{1}$ Rs and $T_{2}$ Rs, sweet, umami, and bitter tastes, consistent with the expression pattern of these GPCRs (Zhang et al., 2003). In contrast, presynaptic (type III) cells respond more broadly to multiple tastants. Information coding within the taste bud is performed by the side-by-side presence of narrowly tuned receptor cells and broadly tuned presynaptic cells.

Presynaptic cells express none of the taste GPCRs nor their downstream effectors, suggesting that they derive at least some of their input indirectly through cell-to-cell signaling. Consistent with this interpretation is the finding that isolated presynaptic cells do not respond to taste qualities mediated by taste GPCRs (Huang et al., 2007). Receptor cells communicate with presynaptic cells during taste stimulation by secreting ATP through Caactivated pannexin 1 hemichannels (Huang et al., 2007). The most parsimonious explanation of the present findings is that, in

den

$\mathrm{SC}$

suc $\underline{\text { Receptor }}$

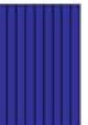

$\vdots 2 \quad 3$
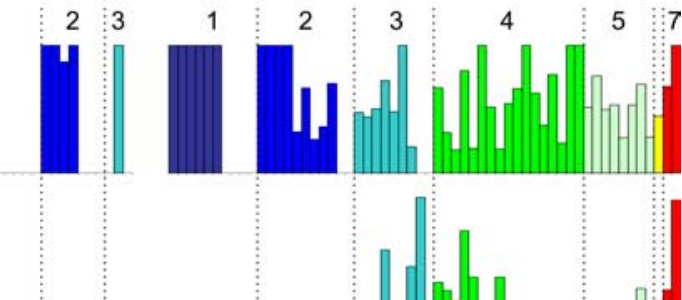

(1)

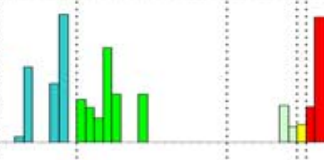

Figure 6. $\quad \mathrm{Ca}^{2+}$ response profiles of receptor and presynaptic cells. Response magnitudes (mean peak $\Delta F / F$ ) of each cell are nitudes are graphed as a percentage of the maximal response for a given cell. Numbers across the top and dotted lines indicate the number of taste stimuli to which each cell responded. The complete raw data are presented in supplemental Tables 1 and 2 (available at www.jneurosci.org as supplemental material). Abbreviations as in Figure 2.

situ, presynaptic cells receive convergent inputs of sweet, umami, and bitter signals from receptor cells.

Although the majority of receptor cells responded narrowly to sweet, bitter, or umami tastes, we occasionally observed some receptor cells responding to two or rarely three taste qualities. There are several possible explanations for this observation. First, occasional receptor cells may express multiple taste receptors. Second, there may be cell-cell communication between receptor cells. Finally, there might be a small degree of uncertainty (noise) in our cell classification.

There appear to be at least two pathways that convey information from taste buds to the CNS. First, receptor cells, by secreting ATP, may pass information directly to afferent nerve fibers that express P2X receptors (Finger et al., 2005; Huang et al., 2007). Mutant mice lacking $\mathrm{P} 2 \mathrm{X}_{2} / \mathrm{P} 2 \mathrm{X}_{3}$ receptors showed reduced behavioral responses to bitter, sweet, and umami tastants but not to sour tastants. Second, we speculate that there is another, parallel pathway involving presynaptic cells. Huang et al. (2006) genetically ablated a category of taste cells that sense sour taste. Bitter, sweet, and umami tastes were unaffected by this ablation. We 


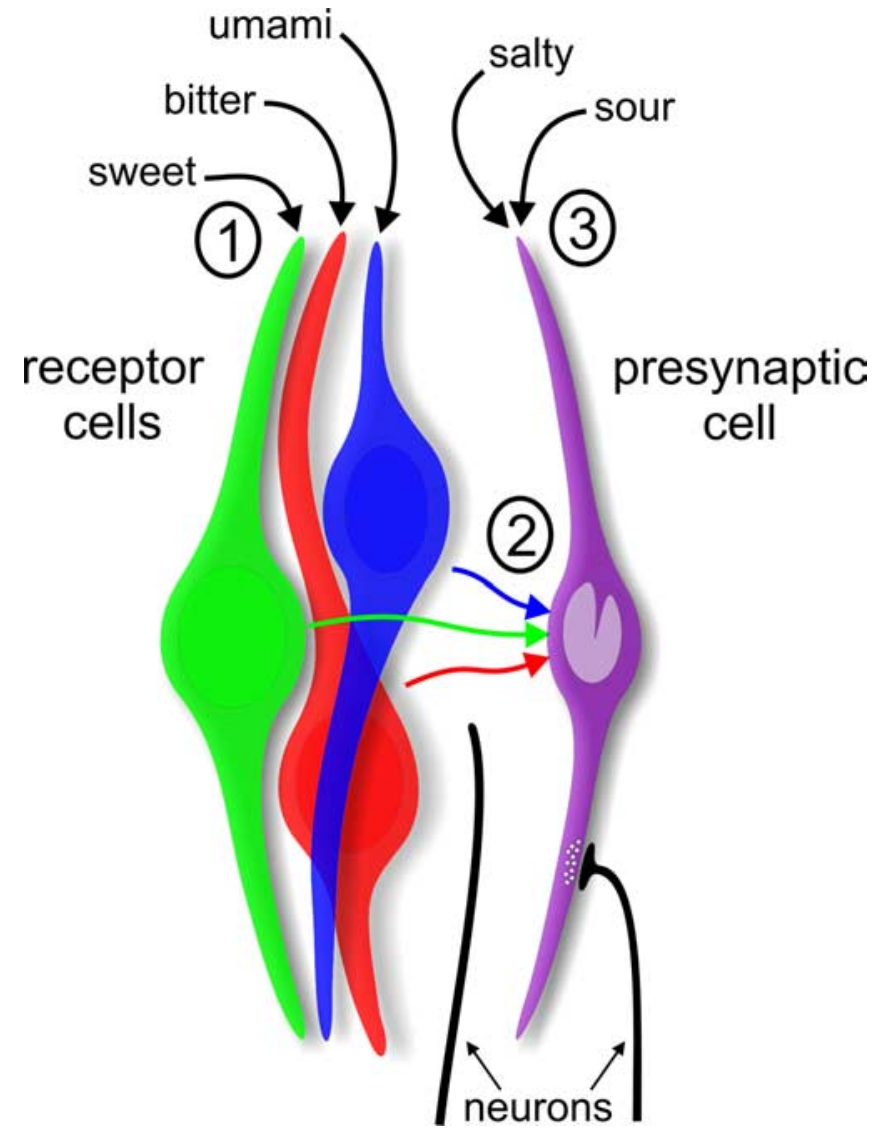

Figure 7. Schematic of gustatory processing based on data from the present study. 1, Receptor (type II) cells are narrowly tuned to sweet, bitter, or umami stimuli. 2, Signals from receptor cells converge onto presynaptic (type III) cells (orange) via purinergic cell-cell communication (cf. Huang et al., 2007). Consequently, presynaptic cells are more broadly responsive than receptor cells to sweet, bitter, and umami stimuli. 3, Furthermore, presynaptic cells also respond to sour and salty tastants, further increasing their breadth of tuning. Primary sensory afferent fibers receive purinergic excitation from receptor cells (Finger et al., 2005; Huang et al., 2007). Presynaptic cells release serotonin (Huang et al., 2007), possibly at the synapses they make with nerve fibers, the origin of which remains unspecified.

report here that sour-sensitive cells are overwhelmingly presynaptic (type III) cells, and thus it is likely that Huang et al. (2006) ablated presynaptic cells. Parenthetically, these authors reported no alteration in gustatory nerve responses evoked by low concentrations of $\mathrm{NaCl}(50-100 \mathrm{~mm})$. Our findings of $\mathrm{NaCl}$ responses in these same cells predict that some aspect of salt taste should be affected by eliminating presynaptic cells. In summary, whether sour- and salt-sensing cells comprise lines of afferent information separate from sweet, bitter, and umami and the identities of transmitters involved in such lines are open questions and remain to be pursued.

Presynaptic cells release serotonin in response to taste stimulation, and thus the synapses that they form and the postulated second pathway may be aminergic (Huang et al., 2005, 2007). Presynaptic cells also express other neurotransmitters. One isoform of glutamic acid decarboxylase, GAD1 (also called GAD67), a biosynthetic enzyme for GABA is expressed in presynaptic cells (DeFazio et al., 2006). Thus, we used GAD-GFP mice to identify presynaptic cells. It is not known whether presynaptic cells release GABA during taste stimulation. Taste-evoked inhibition of afferent neurons has not been extensively studied. Nevertheless, suggestions of taste-evoked inhibitory signals in afferent nerves exist in the literature (Danilova et al., 2002; Frank et al., 2005). Re- cently, prominent inhibition in taste afferents was demonstrated during stimulation with mixtures of sweet and bitter tastants (Frank et al., 2005). Our data suggest that the two components of such mixtures would be primarily detected by different subclasses of receptor cells and would secondarily activate one or more presynaptic cells in the taste bud. The potential role of GABA in mediating these and other inhibitory interactions remains to be explored. Alternatively, GABA in taste buds may function as an excitatory transmitter (Fiumelli and Woodin, 2007) or as a neurotrophic substance (Meier et al., 1999). Unfortunately, GAD1null mice do not live beyond birth (Asada et al., 1997), ruling out taste tests on postnatal animals lacking GABAergic mechanisms. An analysis of the postsynaptic targets of presynaptic cells and the postulated second output pathway, including the neurotransmitter(s) involved, awaits future experimentation.

Insights into how taste cells transmit information to the gustatory sensory afferent neurons can potentially be gleaned by comparing taste cells with responses of individual afferent fibers. Vallate taste buds are innervated by the glossopharyngeal nerve (GL). Previous studies have examined responses of individual afferent fibers in the GL and classified the fibers according to their responses to taste stimulation (for review, see Spector and Travers, 2005). Although there are species-specific differences, several classes of afferents are typically encountered in the GL: N fibers (salt-best), S fibers (sweet-best), Q fibers (quinine/bitterbest), and $\mathrm{H}$ fibers/A fibers (broadly tuned acid- and electrolytesensitive fibers). In addition, umami-best fibers ( $M$ fibers) have been reported in the mouse GL (Ninomiya and Funakoshi, 1989). Our data demonstrate that vallate taste cells have similar tuning to gustatory afferent neurons in the GL. The responses we recorded from receptor (type II) cells are similar to, but more narrowly tuned than, those of $S, Q$, and $M$ fibers. It is therefore possible that sweet-, bitter-, and umami-sensitive receptor cells signal directly to $\mathrm{S}, \mathrm{Q}$, and $\mathrm{M}$ fibers, respectively. Presynaptic (type III) cells respond similarly to $\mathrm{A} / \mathrm{H}$ fibers in at least two ways. First, they are sensitive to acids and are broadly tuned to other tastants (particularly bitter tastants). Second, presynaptic cells frequently have large responses to citric acid and bitter tastants and smaller responses to other taste qualities, in general agreement with the response profiles of A/H fibers. Presynaptic cells form synapses with neurons, which may be $\mathrm{A} / \mathrm{H}$ fibers given the similarities in their response profiles. A recent electrophysiological study similarly found concordance in the response profiles of fungiform taste cells and chorda tympani afferent fibers (Yoshida et al., 2006).

Together, the present data demonstrate clear functional distinctions between receptor (type II) cells and presynaptic (type III) cells. Communication between receptor and presynaptic cells generates more broad responses in taste cells than receptor expression patterns alone would suggest. This communication comprises the first level of information processing in the gustatory system. These results support the notion that the taste bud likely serves a more complex role than simply transmitting signals from molecular receptor to afferent nerve (Spector and Travers, 2005; Roper, 2006).

\section{References}

Asada H, Kawamura Y, Maruyama K, Kume H, Ding RG, Kanbara N, Kuzume H, Sanbo M, Yagi T, Obata K (1997) Cleft palate and decreased brain gamma-aminobutyric acid in mice lacking the $67-\mathrm{kDa}$ isoform of glutamic acid decarboxylase. Proc Natl Acad Sci USA 94:6496-6499.

Caicedo A, Jafri MS, Roper SD (2000) In situ $\mathrm{Ca}^{2+}$ imaging reveals neurotransmitter receptors for glutamate in taste receptor cells. J Neurosci 20:7978-7985. 
Caicedo A, Kim KN, Roper SD (2002) Individual mouse taste cells respond to multiple chemical stimuli. J Physiol (Lond) 544:501-509.

Chandrashekar J, Hoon MA, Ryba NJ, Zuker CS (2006) The receptors and cells for mammalian taste. Nature 444:288-294.

Chattopadhyaya B, Di Cristo G, Higashiyama H, Knott GW, Kuhlman SJ, Welker E, Huang ZJ (2004) Experience and activity-dependent maturation of perisomatic GABAergic innervation in primary visual cortex during a postnatal critical period. J Neurosci 24:9598-9611.

Clapp TR, Yang R, Stoick CL, Kinnamon SC, Kinnamon JC (2004) Morphologic characterization of rat taste Receptor cells that express components of the phospholipase C signaling pathway. J Comp Neurol 468:311-321.

Clapp TR, Medler KF, Damak S, Margolskee RF, Kinnamon SC (2006) Mouse taste cells with G protein-coupled taste receptors lack voltagegated calcium channels and SNAP-25. BMC Biol 4:7.

Danilova V, Danilov Y, Roberts T, Tinti JM, Nofre C, Hellekant G (2002) Sense of taste in a new world monkey, the common marmoset: recordings from the chorda tympani and glossopharyngeal nerves. J Neurophysiol 88:579-594.

DeFazio RA, Dvoryanchikov G, Maruyama Y, Kim JW, Pereira E, Roper SD, Chaudhari N (2006) Separate populations of receptor cells and presynaptic cells in mouse taste buds. J Neurosci 26:3971-3980.

Finger TE, Danilova V, Barrows J, Bartel DL, Vigers AJ, Stone L, Hellekant G, Kinnamon SC (2005) ATP signaling is crucial for communication from taste buds to gustatory nerves. Science 310:1495-1499.

Fiumelli H, Woodin MA (2007) Role of activity-dependent regulation of neuronal chloride homeostasis in development. Curr Opin Neurobiol 17:81-86.

Frank ME, Formaker BK, Hettinger TP (2005) Peripheral gustatory processing of sweet stimuli by golden hamsters. Brain Res Bull 66:70-84.

Gilbertson TA, Boughter Jr JD, Zhang H, Smith DV (2001) Distribution of gustatory sensitivities in rat taste cells: whole-cell responses to apical chemical stimulation. J Neurosci 21:4931-4941.

Herness S, Zhao FL, Kaya N, Shen T, Lu SG, Cao Y (2005) Communication routes within the taste bud by neurotransmitters and neuropeptides. Chem Senses 30 [Suppl 1]:i37-i38.

Huang AL, Chen X, Hoon MA, Chandrashekar J, Guo W, Trankner D, Ryba NJ, Zuker CS (2006) Transient receptor potential family members PKD1L3 and PKD2L1 form a candidate sour taste receptor. Nature 442:934-938.

Huang YJ, Maruyama Y, Lu KS, Pereira E, Plonsky I, Baur JE, Wu D, Roper SD (2005) Mouse taste buds use serotonin as a neurotransmitter. J Neurosci 25:843-847.

Huang YJ, Maruyama Y, Dvoryanchikov G, Pereira E, Chaudhari N, Roper SD (2007) The role of Pannexin 1 hemichannels in ATP release and cell-cell communication in mouse taste buds. Proc Natl Acad Sci USA 104:6436-6441.

Ishimaru $\mathrm{Y}$, Inada $\mathrm{H}$, Kubota $\mathrm{M}$, Zhuang $\mathrm{H}$, Tominaga $\mathrm{M}$, Matsunami $\mathrm{H}$ (2006) Transient receptor potential family members PKD1L3 and PKD2L1 form a candidate sour taste receptor. Proc Natl Acad Sci USA 103:12569-12574.
Kim JW, Roberts C, Maruyama Y, Berg S, Roper S, Chaudhari N (2006) Faithful expression of GFP from the PLC $\beta 2$ promoter in a functional class of taste Receptor cells. Chem Senses 31:213-219.

Lopez-Jimenez ND, Cavenagh MM, Sainz E, Cruz-Ithier MA, Battey JF, Sullivan SL (2006) Two members of the TRPP family of ion channels, Pkd113 and Pkd211, are co-expressed in a subset of taste receptor cells. J Neurochem 98:68-77.

Maruyama Y, Pereira E, Margolskee RF, Chaudhari N, Roper SD (2006) Umami responses in mouse taste cells indicate more than one receptor. J Neurosci 26:2227-2234.

Meier E, Hertz L, Schousboe A (1999) Neurotransmitters as developmental signals. Neurochem Int 19:1-15.

Mueller KL, Hoon MA, Erlenbach I, Chandrashekar J, Zuker CS, Ryba NJ (2005) The receptors and coding logic for bitter taste. Nature 10:225-229.

Nelson G, Hoon MA, Chandrashekar J, Zhang Y, Ryba NJ, Zuker CS (2001) Mammalian sweet taste receptors. Cell 106:381-390.

Ninomiya Y, Funakoshi M (1989) Peripheral neural basis for behavioural discrimination between glutamate and the four basic taste substances in mice. Comp Biochem Physiol 92A:371-376.

Perez CA, Huang L, Rong M, Kozak JA, Preuss AK, Zhang H, Max M, Margolskee RF (2002) A transient receptor potential channel expressed in taste receptor cells. Nat Neurosci 5:1169-1176.

Richter TA, Caicedo A, Roper SD (2003) Sour taste stimuli evoke Ca2+ and pH responses in mouse taste cells. J Physiol (Lond) 547:475-483.

Roper SD (1992) The microphysiology of peripheral taste organs. J Neurosci 12:1127-1134

Roper SD (2006) Cell communication in taste buds. Cell Mol Life Sci 63:1494-1500.

Sato T, Beidler LM (1997) Broad tuning of rat taste cells for four basic taste stimuli. Chem Senses 22:287-293.

Simon SA, de Araujo IE, Gutierrez R, Nicolelis MA (2006) The neural mechanisms of gustation: a distributed processing code. Nat Rev Neurosci 7:890-901.

Smith DV, Travers JB (1979) A metric for the breadth of tuning of gustatory neurons. Chem Senses Flav 4:215-229.

Spector AC, Travers SP (2005) The representation of taste quality in the mammalian nervous system. Behav Cogn Neurosci Rev 4:143-191.

Yang R, Crowley HH, Rock ME, Kinnamon JC (2000) Taste cells with synapses in rat circumvallate papillae display SNAP-25-like immunoreactivity. J Comp Neurol 424:205-215.

Yee CL, Yang R, Bottger B, Finger TE, Kinnamon JC (2001) “Type III” cells of rat taste buds: immunohistochemical and ultrastructural studies of neuron-specific enolase, protein gene product 9.5, and serotonin. J Comp Neurol 440:97-108.

Yoshida R, Shigemura N, Sanematsu K, Yasumatsu K, Ishizuka S, Ninomiya Y (2006) Taste responsiveness of fungiform taste cells with action potentials. J Neurophysiol 96:3088-3095.

Zhang Y, Hoon MA, Chandrashekar J, Mueller KL, Cook B, Wu D, Zuker CS, Ryba NJ (2003) Coding of sweet, bitter, and umami tastes: different receptor cells sharing similar signaling pathways. Cell 112:293-301. 